\title{
Roles of Erythroid Differentiation Regulator 1 (Erdr1) on Inflammatory Skin Diseases
}

\author{
Youn Kyung Houh ${ }^{1,+}$, Kyung Eun Kim ${ }^{2, \dagger}$, Hyun Jeong Park ${ }^{3, *, \dagger}$ and Daeho Cho ${ }^{1,2, *,+}$ \\ 1 Department of Biological Sciences, Sookmyung Women's University, Seoul 04310, Korea; \\ bobada6025@gmail.com \\ 2 Department of Cosmetic Sciences, Sookmyung Women's University, Seoul 04310, Korea; \\ kyungeun@sookmyung.ac.kr \\ 3 Department of Dermatology, Yeouido St. Mary's Hospital, The Catholic University of Korea, \\ Seoul 07345, Korea \\ * Correspondences: hjpark@catholic.ac.kr (H.J.P.); cdhkor@sookmyung.ac.kr (D.C.); \\ Tel.: +82-02-3779-1230 (H.J.P.); +82-02-710-9416 (D.C.) \\ + These authors contributed equally to this work. \\ Academic Editor: Chris Jackson \\ Received: 21 October 2016; Accepted: 5 December 2016; Published: 8 December 2016
}

\begin{abstract}
Erythroid Differentiation Regulator 1 (Erdr1) is known as a hemoglobin synthesis factor which also regulates cell survival under conditions of stress. In addition, previous studies have revealed the effects of Erdr1 on cancer progression and its negative correlation with interleukin (IL)-18, a pro-inflammatory cytokine. Based on this evidence, the therapeutic effects of Erdr1 have been demonstrated in several inflammatory skin diseases such as malignant skin cancer, psoriasis, and rosacea. This article reviews the roles of Erdr1 in skin inflammation, suggesting that Erdr1 is a potential therapeutic molecule on inflammatory disorders.
\end{abstract}

Keywords: Erdr1; IL-18; cutaneous inflammation; melanoma; psoriasis; rosacea

\section{Introduction}

Erythroid Differentiation Regulator 1 (Erdr1) is a recently identified cytokine that was first discovered as a hemoglobin synthesis factor in human and murine erythroleukemia cell lines. Expression of Erdr1, a highly conserved autocrine factor, is widely distributed across many normal mouse and human tissues [1]. Erdr1 is localized at the inner part of cytoplasmic membrane and is secreted through vesicles. Studies have also revealed Erdr1 as a stress-related factor from stroma which controls the survival and growth of hematopoietic progenitors [2]. Erdr1 is secreted immediately from cells in response to several stress conditions. Erdr1 enhances cell survival at low concentration and low cell density but increases cell death at high concentration and high cell density. These opposing effects of Erdr1 on cells are essential for maintaining growth homeostasis.

Recently, Erdr1 has been demonstrated to play additional functions in cancer. This protein regulates the activity of several cancers by inhibiting cell motility and growth. In murine melanoma, Erdr1 overexpression suppressed metastasis in the lung in vivo and inhibited migration, invasion, and proliferation in vitro [3]. Gastric cancer frequently metastasizes to other parts of the body, making it one of the most fatal cancers [4]. Exogenous treatment with recombinant Erdr1 significantly inhibited the migration and invasiveness of gastric cancer by inducing E-cadherin [5]. Additionally, Erdr1 can indirectly repress cancer activity by activating the immune system, specifically enhancing natural killer (NK) cell activation [6]. NK cells are effector lymphocytes which can eliminate tumor cells through cytolytic granule exocytosis and induction of target apoptosis [7]. During NK cell-mediated target cell killing, apoptosis-inducing enzymes such as perforin and granzymes are localized to the tumor 
in the immunological synapse formed between both cells [8]. Lee et al. reported that exogenous treatment of recombinant Erdr1 enhanced the effect of primary human NK cell cytotoxicity of leukemia cancer cells [6]. By regulating actin rearrangement, Erdr1 increases the accumulation of F-actin at the immunological synapse of NK cells. As a result, NK cell cytotoxicity against the target cancer is enhanced. Taken together, Erdr1 can repress cancer progression through inhibition of cancer cell motility and activation of the immune response.

Jung et al. reported that Erdr1 expression is negatively regulated by interleukin (IL)-18, a pro-inflammatory cytokine, in a murine melanoma cell line [3]. In the study, knockdown of IL-18 in B16F10 cells led to increased Erdr1 expression at the RNA and protein levels. The negative correlation between Erdr1 and IL-18 was also confirmed in a human gastric cancer cell line [5]. Both of these cell types have been reported to exhibit a high level of IL-18 expression that is related to cancer progression, including proliferation, metastasis, and angiogenesis [9,10]. However, Erdr1 produces the opposite effect on IL-18 in melanoma and gastric cancer. Due to its role as a pro-inflammatory cytokine, high levels of IL-18 are detected in diseases associated with chronic inflammation [11,12]. Moreover, IL-18 is expressed not only by immune cells but also by non-immune cells like keratinocytes and epithelial cells. In particular, IL-18 plays an important role in cutaneous inflammation $[13,14]$. Therefore, suppression of IL-18 bioactivity can be an effective therapeutic approach for treating chronic inflammatory skin diseases. Accordingly, much research involving Erdr1 has been focused on inflammatory diseases on account of its negative correlation with IL-18.

\section{Roles of Erythroid Differentiation Regulator 1 (Erdr1) on Inflammatory Skin Diseases}

Skin is the largest frontline host defensive tissue against pathogens and consists of two layers. The outer protective barrier, called the epidermis, is composed of keratinocytes, melanocytes, langerhans cells, and merkel cells [15]. The dermis, or lower layer, includes immune cells such as $\mathrm{CD} 4^{+} \mathrm{T}$ cells, $\mathrm{CD} 8^{+} \mathrm{T}$ cells, and macrophages for execution of the immune response upon pathogen invasion $[16,17]$.

As part of its protective function against chemical and physical insults, skin is involved in the innate immune response. Inflammation is one of the key responses of the innate immune system which, along with the adaptive immune response, comprises a complex biological reaction [18]. Injury, infection, and irritation can trigger inflammation in skin tissues, resulting in such common symptoms as heat, pain, redness, and swelling. The purpose of inflammation is to eliminate the initial causes or damaged cells and begin tissue repair. However, long-term inflammation caused by genetic or environmental factors can lead to chronic cutaneous diseases such as psoriasis, rosacea, atopic dermatitis, and alopecia areata, which are associated with uncontrolled immune activity [19]. A variety of cytokines play an important role in the inflammatory pathogenesis of skin. Cutaneous and systemic overexpression of several inflammatory cytokines such as IL-1, IL-6, IL-8, IL-12, IL-18, interferon (IFN)- $\gamma$, and tumor necrosis factor (TNF)- $\alpha$ has been demonstrated $[20,21]$.

It has been reported that uncontrolled production and activation of IL-18 in skin contributes to the pathogenesis of chronic inflammatory diseases [22]. In fact, increased expression of IL-18 and its receptor were observed on lesional skin tissues from patients diagnosed with inflammatory cutaneous diseases such as psoriasis, rosacea, atopic dermatitis, or alopecia areata [19]. Moreover, plasma IL-18 concentration has been linked to the severity of these diseases. Therefore, IL-18 has been considered as a possible marker of various inflammatory skin disorders [23-26].

Erdr1, an IL-18-regulated novel factor, is also expressed in normal skin cells, including keratinocytes and melanocytes [3]. Previous reports revealed that Erdr1 may possibly be associated with skin abnormalities. Kim et al. suggest that Erdr1 is a pro-apoptotic factor in human keratinocytes [27]. Expression of Erdr1 was increased in the HaCaT cell line following ultraviolet B (UVB) irradiation and exogenous treatment of recombinant Erdr1 enhanced UVB-induced apoptosis via caspase-3 activation. 
Here, we review recent findings that highlight the correlation between Erdr1, an IL-18-regulated factor, and chronic inflammation in cutaneous disorders such as melanoma, psoriasis, and rosacea.

\subsection{Effects of Erdr1 on Malignant Skin Cancer and Melanoma}

Melanoma is one type of malignant skin tumor that arises from melanocytes localized in the epidermal skin layer [28]. Chronic inflammation has been suggested as one of the hallmarks of exposure to ultraviolet (UV) radiation and other environmental factors that cause skin cancers [29]. Recently, much molecular evidence that is focused primarily on the role of inflammation in melanoma has been accumulating. These data include the role of TNF, IL-1, IL-6, IL-18, matrix metalloproteases (MMP), and vascular endothelial growth factor (VEGF), which are NF-kB-regulated inflammatory factors predominantly expressed in cancer. In addition, the activity of NF- $\mathrm{kB}$ is usually regulated by other transcription factors such as signal transducer and activator of transcription 3 (STAT3) [30].

Jung et al. found that Erdr1 expression was higher in normal melanocytes compared with melanoma cell lines. This result was also confirmed in human skin tissues from melanoma patients and healthy donors, indicating that Erdr1 expression is downregulated in melanoma. In the study, Erdr1 expression was found to be negatively regulated by IL-18, which has a pro-cancer effect on melanoma [3]. Overexpression of Erdr1 significantly inhibited cell migration, invasion, and proliferation in B16F10, a murine melanoma, in vitro. In addition, melanoma lung metastasis and tumor growth were suppressed in mice implanted with Erdr1-overexpressing melanoma. This study found that Erdr1 overexpression leads to the downregulation of heat shock protein 90 (HSP90), a ubiquitous chaperone that reportedly acts as a pro-cancer factor which is enhanced in advanced malignant melanoma related to stress conditions. Studies have established HSP90 as a marker of progression in melanoma and a therapeutic target in cancer [31,32]. Inhibition of HSP90 suggests that Erdr1 acts as a regulatory protein for melanoma motility. Moreover, HSP90 regulates NF- $\mathrm{kB}$ signaling, an important pathway involved in mediating the inflammatory response [33]. In fact, HSP90 inhibitors have been shown to attenuate inflammatory responses, suggesting that HSP90 could be a therapeutic target for treating various inflammatory skin disorders [34,35].

Melanoma is resistant to diverse conventional chemotherapy due to its aggressiveness [36]. Therefore, development of an effective therapeutic strategy involving apoptosis of cancer cells is necessary. Lee et al. showed that Erdr1 could suppress murine melanoma growth through the regulation of apoptosis in vitro and in vivo [37]. Intraperitoneal injection of recombinant Erdr1 significantly reduced melanoma growth implanted onto mouse skin. Decreased B-cell lymphoma 2 (Bcl-2) and increased bcl-2 like protein 4 (Bax) expression were detected on tumor tissues from the affected mouse. Moreover, exogenous treatment of recombinant Erdr1 on B16F10 cells increased apoptosis by reducing Bcl-2 expression while enhancing Bax in vitro. High expression of Bcl-2, an anti-apoptotic factor, has been associated with resistance to chemotherapy in human melanoma and other tumor types [38]. Another Bcl-2 family member, Bax, exhibits pro-apoptotic effects, thereby opposing Bcl-2. Clinical trials for treating melanoma that target the Bcl-2 family are important because these proteins act as regulators of the mitochondrial apoptotic pathway $[39,40]$. Activity of the well-established apoptosis-related signaling molecule STAT3 was also inhibited by exogenous Erdr1 [41]. As mentioned, activation of STAT3 mediates tumor-promoting inflammation and can regulate the critical transcription factor, NF- $\mathrm{kB}$. Thus, downregulation of STAT3 activity has been considered a promising strategy for treating inflammatory diseases and cancer [42].

The relationship between melanoma and IL-18 has been widely studied. Studies have demonstrated that IL-18 enhances the migration of murine melanoma cells via the generation of "reactive oxygen species" (ROS) and the MAPK pathway [43]. Metastatic patients showed significantly higher IL-18 expression than both healthy controls and non-metastatic patients [44,45]. Therefore, an IL-18R-expressing phenotype may be a diagnostic biomarker indicating predisposition of melanoma metastasis $[45,46]$. Melanomas developed through inflammation-dependent mechanisms have high metastatic potential that is dependent on IL-18, which can activate the STAT3 pathway. 
Taken together, the negative correlation between Erdr1 and IL-18 suggests a potential anti-inflammatory effect that occurs through regulation of inflammatory response-related molecules such as HSP90 and STAT3, which are also candidates to be targeted for melanoma therapy (Figure 1).

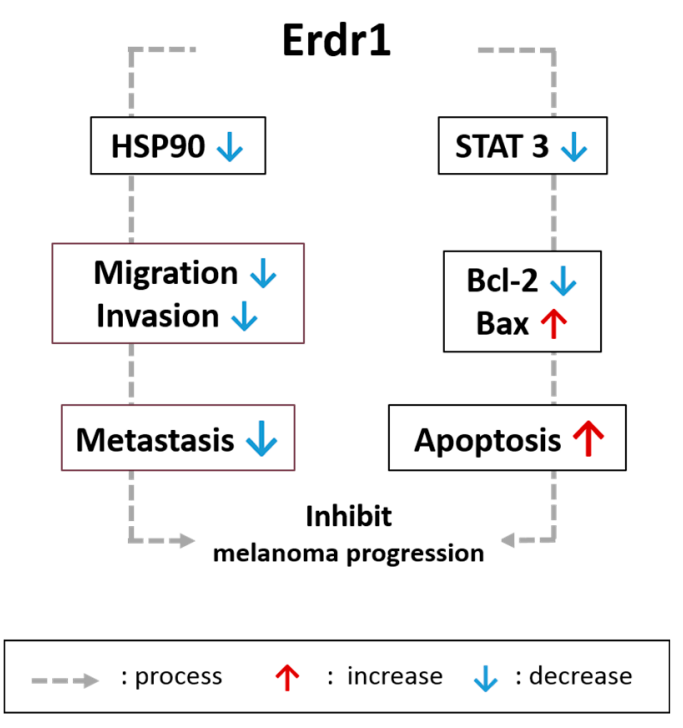

Figure 1. Erdr1 inhibits melanoma progression. Erdr1 suppresses metastasis by downregulating migration and invasion ability via HSP90 inhibition. In addition, Erdr1 reduces the apoptosis-regulating STAT3 signaling pathway. Erdr1 exerts its anti-cancerous effects by regulating HSP90 and STAT3 in melanoma.

\subsection{Roles of Erdr1 on Psoriasis}

Psoriasis is a common chronic skin disease that affects $2 \%-3 \%$ of the global population and is considered an immune-mediated inflammatory disorder. Psoriatic skin lesions are characterized by the manifestation of red and white scaly plaques on the top layer [47]. Environmental (stress, microorganisms, drugs, trauma, smoking, etc.) and genetic factors initiate stressor (LL-37) production from epidermal keratinocytes [48,49]. They activate myeloid dendritic cells to induce the differentiation of naïve T cells into effector cells such as Th1 and Th17 cells [50]. These effector cells expressing the specific chemokine receptors escape from blood vessels and migrate into skin tissue along chemokine gradients. Then, the cells secrete several pro-inflammatory cytokines, such as interferon- $\gamma$, TNF- $\alpha$, IL-17, and IL-22, for keratinocytes to respond. Recent research has been focused on IL-17-producing Th17 cells. Therefore, this response is unique to epithelial immunity which bridges the adaptive immune response and keratinocyte dysregulation in psoriasis [51].

Erdr1 has been suggested to act as a stress-related factor that regulates cell growth and survival [2]. Kim et al. first reported that Erdr1 may be associated with skin abnormalities [27]. In the skin, UVB irradiation is a common stressful condition which causes DNA damage and leads to apoptosis [52,53]. Enhancement of both Erdr1 mRNA and protein expression were enhanced by UVB irradiation depending on Erk and p38 MAPK phosphorylation in HaCaT, a human keratinocyte cell line [54]. Moreover, UVB-mediated apoptosis was reduced in Erdr1 knockdown cells whereas apoptosis was increased in Erdr1-overexpressing cells. These data demonstrate that apoptosis induced by UVB is dependent on Erdr1 expression in keratinocytes. UVB irradiation is a very harmful biological stressor, so a better outcome for cells is to undergo suicide via apoptosis rather than permanent mutation. Therefore, as a pro-apoptotic factor, Erdr1 could be a therapeutic molecule for treating skin diseases, such as skin cancer and psoriasis, which are characterized by low apoptosis and uncontrolled cell proliferation.

Keratinocytes stimulated by various genetic predispositions or exogenous triggers exhibit hyper-proliferation and aberrant differentiation, releasing antimicrobial peptides as well as chemokines 
and inflammatory cytokines including IL-18 [55-57]. In fact, IL-18 levels are significantly higher in skin lesions of psoriasis patients relative to healthy controls [58]. Because serum IL-18 concentration is linked to psoriasis severity (PASI), IL-18 might be considered as a possible biomarker of psoriasis [24]. In HaCaT cells, Erdr1 expression is also inversely correlated with IL-18 [59]. Kim et al. compared Erdr1 expression in skin tissues from human psoriasis patients and healthy volunteers. Histological analysis by immunohistochemistry staining suggested that Erdr1 was significantly downregulated in psoriatic lesional skin compared to healthy skin. Meanwhile, IL-18 expression exhibited the opposite expression pattern. Moreover, after effective therapeutic treatment using cyclosporine A in a psoriasis-like mouse model, the reduced Erdr1 mediated by induction of psoriasis-like skin inflammation was reversed and symptoms were relieved [60]. Intraperitoneal administration of recombinant Erdr1 exerted a therapeutic effect on a psoriasis-like mouse model which was induced by imiquimod via activating the IL-23/IL-17 axis [61]. Erdr1 significantly improved redness, thickness and prevalence of scales of lesional skin of a psoriasis-like skin inflammatory mouse model. Furthermore, keratinocyte acanthosis, parakeratosis, and desquamation, as well as inflammatory infiltration, were alleviated in psoriasis-like lesions. Various biomarkers for psoriasis, such as keratins $6,14,16$, and S100A8, were reduced by Erdr1. Major inflammatory cytokines such as IL-17, IL-22, and TNF- $\alpha$ are also reduced by Erdr1 administration. Additionally, Erdr1 administration inhibited CCR $6^{+}$cell infiltration into epidermal sites by blocking the production of CCL20, a chemokine for CCR6 ${ }^{+}$cells, from keratinocytes [60]. In psoriasis pathogenesis, $\mathrm{CCR} 6^{+}$Th17 cells are highly accumulated in lesional skin, stimulating keratinocytes through IL-17 production [62]. Therefore, the result suggest that Erdr1 reduces CCR6 ${ }^{+}$Th17 migration toward the lesional site. Taken together, Erdr1 may be involved in psoriasis pathogenesis, making it a potential biomarker and therapeutic molecule (Figure 2).

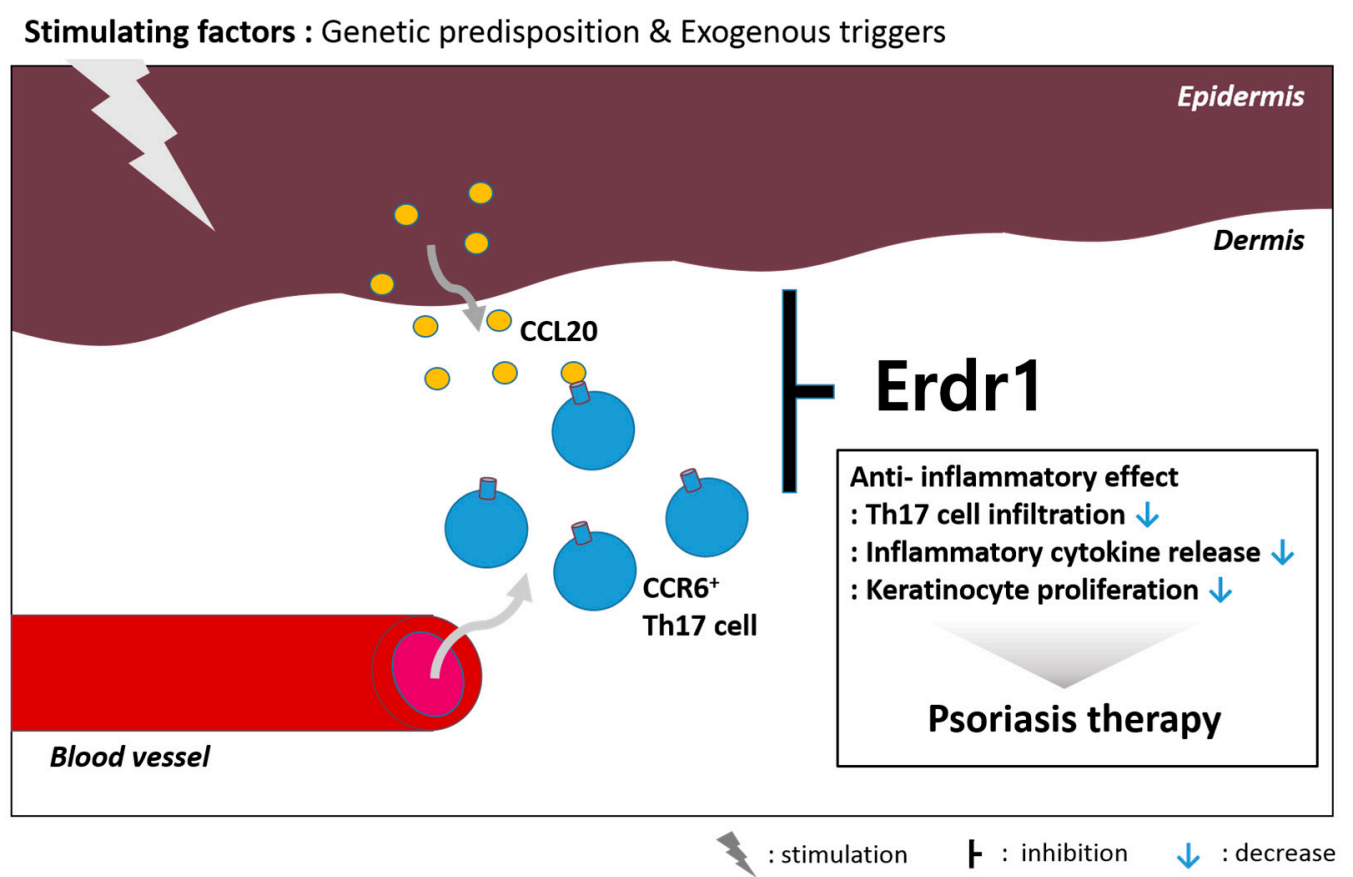

Figure 2. Erdr1 is a potential therapeutic molecule for psoriasis due to its role in suppressing inflammation. Genetic and environmental triggers stimulate the epidermis, which produces chemokines for inflammatory immune cell infiltration. More specifically, Erdr1 inhibits the infiltration of CCR6 ${ }^{+}$Th17 cells via blockade of CCL20.

\subsection{Therapeutinc Effect of Erdr1 on Rosacea}

Besides psoriasis, rosacea is a common chronic inflammatory skin disease which mainly affects the central facial skin tissue, including the forehead, nose, chin, and cheeks [63]. The pathology of 
this disease results from very complex communication between the cutaneous vascular, nervous, and immune system in the skin [63-65]. Angiogenesis is a characteristic of rosacea and plays a critical role in the inflammatory response, leading to exacerbation of rosacea pathogenesis. Angiogenesis is vital for many physiological and pathological reactions, including wound healing, inflammation, tumor growth, and metastatic spread. In particular, VEGF, which is known as a major skin angiogenesis factor, is released by epidermal keratinocytes on lesional rosacea tissues [66].

Trigger factors such as LL-37 stimulate the innate immune response and cause neurovascular dysfunction, leading to vasodilation, inflammation, fibrosis, and neurosensory system activation. An altered innate immune response results in both inflammation and angiogenesis [67]. In rosacea skin, perivascular infiltration and inflammation can be observed throughout the upper to deep dermis. Erdr1 expression has been examined in the skin tissues of patients with rosacea [68]. Histological analysis revealed that Erdr1 expression is lower in patients with rosacea than normal controls. However, IL-18 exhibited a higher expression level in rosacea patients compared to healthy donors.

In addition, a therapeutic effect of Erdr1 on a rosacea-like mouse model involving the regulation of angiogenesis was identified. Intraperitoneal administration of recombinant Erdr1 relieved clinical rosacea symptoms in an LL-37-induced rosacea mouse model. Decreased VEGF expression and suppressed $\mathrm{CD}_{3} 4^{+}$microvessels were also detected on the skin tissues of Erdr1-treated animals, suggesting that Erdr1 suppresses angiogenesis. Additionally, an anti-inflammatory effect of Erdr1 mediated by reduced infiltration of inflammatory cells such as $\mathrm{CD}^{+}$and $\mathrm{CD} 8^{+} \mathrm{T}$ cells was demonstrated. These data suggest that Erdr1 suppresses T cell-mediated inflammation (Figure 3).

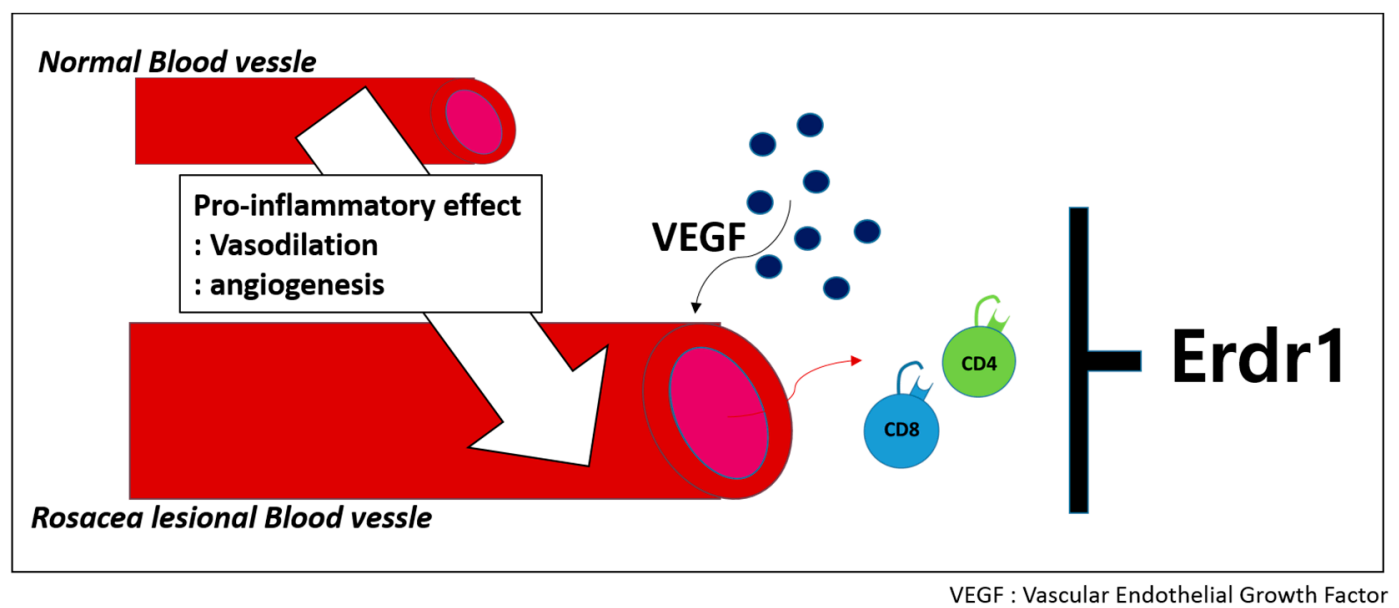

Figure 3. Erdr1 is suggested as a candidate target for rosacea treatment due to its anti-inflammatory and anti-angiogenic effects.

Studies have suggested that IL-18 is an angiogenic mediator due to its ability to induce endothelial tube formation in vitro and in vivo [69]. Enhanced expression of VEGF family members and their receptors was detected in the skin of rosacea patients, suggesting that VEGF is a potential therapeutic target through inhibiting angiogenesis $[67,70]$. Several therapeutic agents which decrease inflammation and control angiogenesis via inhibition of VEGF production and neovessel formation have been introduced to treat rosacea [71,72]. Overall, these data suggest that Erdr1 can be an effective therapeutic candidate via its anti-inflammatory and anti-angiogenic effects.

\section{Conclusions}

This article reviews the effects of Erdr1 on inflammatory skin diseases. It has been reported that Erdr1 overexpression suppressed melanoma metastasis by reducing HSP90 expression. HSP90 has a pro-inflammatory effect as well as a pro-cancerous one, suggesting that HSP90 could be a therapeutic target for treating inflammatory diseases, especially in psoriasis. In fact, HSP90 inhibitor alleviated 
psoriasis-like skin inflammation in a mouse model [35]. Additionally, recombinant Erdr1 treatment reduced STAT3 activity in melanoma cell line. STAT3 is known as a critical signaling molecule involved in cancer and inflammation. Epidermal keratinocytes in psoriatic lesions are characterized by activated STAT3. STAT3 inhibitors markedly improved psoriasis symptoms in patients [73]. Taken together, Erdr1 exerts a therapeutic effect on the inflammatory skin diseases due to its overall anti-inflammatory effects. Besides skin diseases, Erdr1 can be a potential therapeutic molecule for other disorders that are characterized by inflammation.

Acknowledgments: This research was supported by Creative Materials Discovery Program through the National Research Foundation of Korea (NRF) funded by the Ministry of Science, ICT and Future Planning (2016M3D1A1021387) and Basic Science Research Program through the NRF funded by the Ministry of Education, Science and Technology (NRF-2013R1A1A2062797).

Author Contributions: Youn Kyung Houh and Kyung Eun Kim wrote the manuscript. Daeho Cho and Hyun Jeong Park designed the review and assisted to write the manuscript.

Conflicts of Interest: The authors declare no conflict of interest.

\section{References}

1. Dormer, P.; Spitzer, E.; Frankenberger, M.; Kremmer, E. Erythroid differentiation regulator (Edr), a novel, highly conserved factor I. Induction of haemoglobin synthesis in erythroleukaemic cells. Cytokine 2004, 26, 231-242. [CrossRef] [PubMed]

2. Dormer, P.; Spitzer, E.; Moller, W. Edr is a stress-related survival factor from stroma and other tissues acting on early haematopoietic progenitors (E-Mix). Cytokine 2004, 27, 47-57. [CrossRef] [PubMed]

3. Jung, M.K.; Park, Y.; Song, S.B.; Cheon, S.Y.; Park, S.; Houh, Y.; Ha, S.; Kim, H.J.; Park, J.M.; Kim, T.S.; et al. Erythroid differentiation regulator 1, an interleukin 18-regulated gene, acts as a metastasis suppressor in melanoma. J. Investig. Dermatol. 2011, 131, 2096-2104. [CrossRef] [PubMed]

4. Hu, B.; El Hajj, N.; Sittler, S.; Lammert, N.; Barnes, R.; Meloni-Ehrig, A. Gastric cancer: Classification, histology and application of molecular pathology. J. Gastrointest. Oncol. 2012, 3, 251-261. [PubMed]

5. Jung, M.K.; Houh, Y.K.; Ha, S.; Yang, Y.; Kim, D.; Kim, T.S.; Yoon, S.R.; Bang, S.I.; Cho, B.J.; Lee, W.J.; et al. Recombinant Erdr1 suppresses the migration and invasion ability of human gastric cancer cells, SNU-216, through the JNK pathway. Immunol. Lett. 2013, 150, 145-151. [CrossRef] [PubMed]

6. Lee, H.R.; Huh, S.Y.; Hur, D.Y.; Jeong, H.; Kim, T.S.; Kim, S.Y.; Park, S.B.; Yang, Y.; Bang, S.I.; Park, H.; et al. Erdr1 enhances human NK cell cytotoxicity through an actin-regulated degranulation-dependent pathway. Cell. Immunol. 2014, 292, 78-84. [CrossRef] [PubMed]

7. Roda-Navarro, P.; Reyburn, H.T. Intercellular protein transfer at the NK cell immune synapse: Mechanisms and physiological significance. FASEB J. 2007, 21, 1636-1646. [CrossRef] [PubMed]

8. Davis, D.M.; Chiu, I.; Fassett, M.; Cohen, G.B.; Mandelboim, O.; Strominger, J.L. The human natural killer cell immune synapse. Proc. Natl. Acad. Sci. USA 1999, 96, 15062-15067. [CrossRef] [PubMed]

9. Vidal-Vanaclocha, F.; Fantuzzi, G.; Mendoza, L.; Fuentes, A.M.; Anasagasti, M.J.; Martin, J.; Carrascal, T.; Walsh, P.; Reznikov, L.L.; Kim, S.H.; et al. IL-18 regulates IL-1 $\beta$-dependent hepatic melanoma metastasis via vascular cell adhesion molecule-1. Proc. Natl. Acad. Sci. USA 2000, 97, 734-739. [CrossRef] [PubMed]

10. Kang, J.S.; Bae, S.Y.; Kim, H.R.; Kim, Y.S.; Kim, D.J.; Cho, B.J.; Yang, H.K.; Hwang, Y.I.; Kim, K.J.; Park, H.S.; et al. Interleukin-18 increases metastasis and immune escape of stomach cancer via the downregulation of CD70 and maintenance of CD44. Carcinogenesis 2009, 30, 1987-1996. [CrossRef] [PubMed]

11. Keyel, P.A. How is inflammation initiated? Individual influences of IL-1, IL-18 and HMGB1. Cytokine 2014, 69, 136-145. [CrossRef] [PubMed]

12. Sedimbi, S.K.; Hagglof, T.; Karlsson, M.C. IL-18 in inflammatory and autoimmune disease. Cell. Mol. Life Sci. 2013, 70, 4795-4808. [CrossRef] [PubMed]

13. Okamura, H.; Tsutsi, H.; Komatsu, T.; Yutsudo, M.; Hakura, A.; Tanimoto, T.; Torigoe, K.; Okura, T.; Nukada, Y.; Hattori, K.; et al. Cloning of a new cytokine that induces IFN- $\gamma$ production by T cells. Nature 1995, 378, 88-91. [CrossRef] [PubMed]

14. Dinarello, C.A. Interleukin-18, a proinflammatory cytokine. Eur. Cytokine Netw. 2000, 11, 483-486. [PubMed]

15. Pasparakis, M.; Haase, I.; Nestle, F.O. Mechanisms regulating skin immunity and inflammation. Nat. Rev. Immunol. 2014, 14, 289-301. [CrossRef] [PubMed] 
16. Di Meglio, P.; Perera, G.K.; Nestle, F.O. The multitasking organ: Recent insights into skin immune function. Immunity 2011, 35, 857-869. [CrossRef] [PubMed]

17. Nestle, F.O.; di Meglio, P.; Qin, J.Z.; Nickoloff, B.J. Skin immune sentinels in health and disease. Nat. Rev. Immunol. 2009, 9, 679-691. [CrossRef] [PubMed]

18. Aggarwal, B.B.; Shishodia, S.; Sandur, S.K.; Pandey, M.K.; Sethi, G. Inflammation and cancer: How hot is the link? Biochem. Pharmacol. 2006, 72, 1605-1621. [CrossRef] [PubMed]

19. Lee, J.H.; Cho, D.H.; Park, H.J. IL-18 and cutaneous inflammatory diseases. Int. J. Mol. Sci. 2015, 16, 29357-29369. [CrossRef] [PubMed]

20. Nedoszytko, B.; Sokolowska-Wojdylo, M.; Ruckemann-Dziurdzinska, K.; Roszkiewicz, J.; Nowicki, R.J. Chemokines and cytokines network in the pathogenesis of the inflammatory skin diseases: Atopic dermatitis, psoriasis and skin mastocytosis. Postep. Derm. Alergol. 2014, 31, 84-91. [CrossRef] [PubMed]

21. Sauder, D.N. The role of epidermal cytokines in inflammatory skin diseases. J. Investig. Dermatol. 1990, 95, 27S-28S. [CrossRef] [PubMed]

22. Boraschi, D.; Dinarello, C.A. IL-18 in autoimmunity: Review. Eur. Cytokine Netw. 2006, 17, 224-252. [PubMed]

23. Pietrzak, A.; Janowski, K.; Chodorowska, G.; Michalak-Stoma, A.; Rolinski, J.; Zalewska, A.; Jastrzebska, I.; Tabarkiewicz, J.; Paszkowski, T.; Kapec, E.; et al. Plasma interleukin-18 and dendritic cells in males with psoriasis vulgaris. Mediators Inflamm. 2007, 2007, 61254. [CrossRef] [PubMed]

24. Flisiak, I.; Klepacki, A.; Chodynicka, B. Plasma and scales levels of interleukin 18 in comparison with other possible clinical and laboratory biomarkers of psoriasis activity. Biomarkers 2006, 11, 194-200. [CrossRef] [PubMed]

25. Hon, K.L.; Leung, T.F.; Ma, K.C.; Wong, C.K.; Wan, H.; Lam, C.W. Serum concentration of IL-18 correlates with disease extent in young children with atopic dermatitis. Pediatr. Dermatol. 2004, 21, 619-622. [CrossRef] [PubMed]

26. Puxeddu, I.; Italiani, P.; Giungato, P.; Pratesi, F.; Panza, F.; Bartaloni, D.; Rocchi, V.; del Corso, I.; Boraschi, D.; Migliorini, P. Free IL-18 and IL-33 cytokines in chronic spontaneous urticaria. Cytokine 2013, 61, 741-743. [CrossRef] [PubMed]

27. Kim, H.J.; Song, S.B.; Yang, Y.; Eun, Y.S.; Cho, B.K.; Park, H.J.; Cho, D.H. Erythroid differentiation regulator 1 (Erdr1) is a proapototic factor in human keratinocytes. Exp. Dermatol. 2011, 20, 920-925. [CrossRef] [PubMed]

28. Mancianti, M.L.; Herlyn, M. Tumor progression in melanoma: The biology of epidermal melanocytes in vitro. Carcinogenesis 1989, 11, 369-386.

29. Maru, G.B.; Gandhi, K.; Ramchandani, A.; Kumar, G. The role of inflammation in skin cancer. Adv. Exp. Med. Biol. 2014, 816, 437-469. [PubMed]

30. Kraus, S.; Arber, N. Inflammation and colorectal cancer. Curr. Opin. Pharmacol. 2009, 9, 405-410. [CrossRef] [PubMed]

31. McCarthy, M.M.; Pick, E.; Kluger, Y.; Gould-Rothberg, B.; Lazova, R.; Camp, R.L.; Rimm, D.L.; Kluger, H.M. HSP90 as a marker of progression in melanoma. Ann. Oncol. 2008, 19, 590-594. [CrossRef] [PubMed]

32. Soo, E.T.; Yip, G.W.; Lwin, Z.M.; Kumar, S.D.; Bay, B.H. Heat shock proteins as novel therapeutic targets in cancer. In Vivo 2008, 22, 311-315. [PubMed]

33. Sevin, M.; Girodon, F.; Garrido, C.; de Thonel, A. HSP90 and HSP70: Implication in inflammation processes and therapeutic approaches for myeloproliferative neoplasms. Mediators Inflamm. 2015, 2015, 970242. [CrossRef] [PubMed]

34. Madrigal-Matute, J.; Lopez-Franco, O.; Blanco-Colio, L.M.; Munoz-Garcia, B.; Ramos-Mozo, P.; Ortega, L.; Egido, J.; Martin-Ventura, J.L. Heat shock protein 90 inhibitors attenuate inflammatory responses in atherosclerosis. Cardiovasc. Res. 2010, 86, 330-337. [CrossRef] [PubMed]

35. Stenderup, K.; Rosada, C.; Gavillet, B.; Vuagniaux, G.; Dam, T.N. DEBIO 0932, a new oral HSP90 inhibitor, alleviates psoriasis in a xenograft transplantation model. Acta Derm. Venereol. 2014, 94, 672-676. [CrossRef] [PubMed]

36. Soengas, M.S.; Lowe, S.W. Apoptosis and melanoma chemoresistance. Oncogene 2003, 22, 3138-3151. [CrossRef] [PubMed]

37. Lee, J.; Jung, M.K.; Park, H.J.; Kim, K.E.; Cho, D. Erdr1 suppresses murine melanoma growth via regulation of apoptosis. Int. J. Mol. Sci. 2016, 17, 107. [CrossRef] [PubMed] 
38. Helmbach, H.; Rossmann, E.; Kern, M.A.; Schadendorf, D. Drug-resistance in human melanoma. Int. J. Cancer 2001, 93, 617-622. [CrossRef] [PubMed]

39. Del Bufalo, D.; Trisciuoglio, D.; Scarsella, M.; Zangemeister-Wittke, U.; Zupi, G. Treatment of melanoma cells with a Bcl-2/Bcl-xl antisense oligonucleotide induces antiangiogenic activity. Oncogene 2003, 22, 8441-8447. [CrossRef] [PubMed]

40. Piro, L.D. Apoptosis, Bcl-2 antisense, and cancer therapy. Oncology 2004, 18, 5-10. [PubMed]

41. Fofaria, N.M.; Srivastava, S.K. Critical role of STAT3 in melanoma metastasis through anoikis resistance. Oncotarget 2014, 5, 7051-7064. [CrossRef] [PubMed]

42. Yu, H.; Pardoll, D.; Jove, R. STATS in cancer inflammation and immunity: A leading role for STAT3. Nat. Rev. Cancer 2009, 9, 798-809. [CrossRef] [PubMed]

43. Jung, M.K.; Song, H.K.; Kim, K.E.; Hur, D.Y.; Kim, T.; Bang, S.; Park, H.; Cho, D.H. IL-18 enhances the migration ability of murine melanoma cells through the generation of ROI and the MAPK pathway. Immunol. Lett. 2006, 107, 125-130. [CrossRef] [PubMed]

44. Lissoni, P.; Brivio, F.; Rovelli, F.; Fumagalli, G.; Malugani, F.; Vaghi, M.; Secondino, S.; Bucovec, R.; Gardani, G.S. Serum concentrations of interleukin-18 in early and advanced cancer patients: Enhanced secretion in metastatic disease. J. Biol. Regul. Homeost. Agents 2000, 14, 275-277. [PubMed]

45. Crende, O.; Sabatino, M.; Valcarcel, M.; Carrascal, T.; Riestra, P.; Lopez-Guerrero, J.A.; Nagore, E.; Mandruzzato, S.; Wang, E.; Marincola, F.M.; et al. Metastatic lesions with and without interleukin-18dependent genes in advanced-stage melanoma patients. Am. J. Pathol. 2013, 183, 69-82. [CrossRef] [PubMed]

46. Park, H.; Byun, D.; Kim, T.S.; Kim, Y.I.; Kang, J.S.; Hahm, E.S.; Kim, S.H.; Lee, W.J.; Song, H.K.; Yoon, D.Y.; et al. Enhanced IL-18 expression in common skin tumors. Immunol. Lett. 2001, 79, 215-219. [CrossRef]

47. Wagner, E.F.; Schonthaler, H.B.; Guinea-Viniegra, J.; Tschachler, E. Psoriasis: What we have learned from mouse models. Nat. Rev. Rheumatol. 2010, 6, 704-714. [CrossRef] [PubMed]

48. Capon, F.; di Meglio, P.; Szaub, J.; Prescott, N.J.; Dunster, C.; Baumber, L.; Timms, K.; Gutin, A.; Abkevic, V.; Burden, A.D.; et al. Sequence variants in the genes for the interleukin-23 receptor (IL23R) and its ligand (IL12B) confer protection against psoriasis. Hum. Genet. 2007, 122, 201-206. [CrossRef] [PubMed]

49. Nestle, F.O.; Kaplan, D.H.; Barker, J. Psoriasis. N. Engl. J. Med. 2009, 361, 496-509. [CrossRef] [PubMed]

50. Nestle, F.O.; Turka, L.A.; Nickoloff, B.J. Characterization of dermal dendritic cells in psoriasis. Autostimulation of T lymphocytes and induction of Th1 type cytokines. J. Clin. Investig. 1994, 94, 202-209. [CrossRef] [PubMed]

51. Zheng, Y.; Danilenko, D.M.; Valdez, P.; Kasman, I.; Eastham-Anderson, J.; Wu, J.; Ouyang, W. Interleukin-22, a $\mathrm{T}_{\mathrm{H}} 17$ cytokine, mediates IL-23-induced dermal inflammation and acanthosis. Nature 2007, 445, 648-651. [CrossRef] [PubMed]

52. Cleaver, J.E.; Crowley, E. Uv damage, DNA repair and skin carcinogenesis. Front. Biosci. 2002, 7, d1024-d1043. [CrossRef] [PubMed]

53. Sarasin, A. The molecular pathways of ultraviolet-induced carcinogenesis. Mutat. Res. 1999, 428, 5-10. [CrossRef]

54. Albanesi, C.; Scarponi, C.; Giustizieri, M.L.; Girolomoni, G. Keratinocytes in inflammatory skin diseases. Curr. Drug. Targets Inflamm. Allergy 2005, 4, 329-334. [CrossRef] [PubMed]

55. Griffiths, C.E.; Barker, J.N. Pathogenesis and clinical features of psoriasis. Lancet 2007, 370, $263-271$. [CrossRef]

56. Bos, J.D.; de Rie, M.A. The pathogenesis of psoriasis: Immunological facts and speculations. Immunol. Today 1999, 20, 40-46. [CrossRef]

57. Ohta, Y.; Hamada, Y.; Katsuoka, K. Expression of IL-18 in psoriasis. Arch. Dermatol. Res. 2001, 293, 334-342. [CrossRef] [PubMed]

58. Arican, O.; Aral, M.; Sasmaz, S.; Ciragil, P. Serum levels of TNF- $\alpha$, IFN- $\gamma$, IL-6, IL-8, IL-12, IL-17, and IL-18 in patients with active psoriasis and correlation with disease severity. Mediators Inflamm. 2005, 2005, $273-279$. [CrossRef] [PubMed]

59. Kim, K.E.; Houh, Y.; Lee, J.; Kim, S.; Cho, D.; Park, H.J. Downregulation of erythroid differentiation regulator 1 (Erdr1) plays a critical role in psoriasis pathogenesis. Exp. Dermatol. 2016, 25, 570-572. [CrossRef] [PubMed]

60. Kim, K.E.; Houh, Y.; Park, H.J.; Cho, D. Therapeutic effects of erythroid differentiation regulator 1 on imiquimod-induced psoriasis-like skin inflammation. Int. J. Mol. Sci. 2016, 17, 244. [CrossRef] [PubMed] 
61. Van der Fits, L.; Mourits, S.; Voerman, J.S.; Kant, M.; Boon, L.; Laman, J.D.; Cornelissen, F.; Mus, A.M.; Florencia, E.; Prens, E.P.; et al. Imiquimod-induced psoriasis-like skin inflammation in mice is mediated via the IL-23/IL-17 axis. J. Immunol. 2009, 182, 5836-5845. [CrossRef] [PubMed]

62. Yamazaki, T.; Yang, X.O.; Chung, Y.; Fukunaga, A.; Nurieva, R.; Pappu, B.; Martin-Orozco, N.; Kang, H.S.; Ma, L.; Panopoulos, A.D.; et al. CCR6 regulates the migration of inflammatory and regulatory $\mathrm{T}$ cells. J. Immunol. 2008, 181, 8391-8401. [CrossRef] [PubMed]

63. Steinhoff, M.; Schauber, J.; Leyden, J.J. New insights into rosacea pathophysiology: A review of recent findings. J. Am. Acad. Dermatol. 2013, 69, S15-S26. [CrossRef] [PubMed]

64. Steinhoff, M.; Buddenkotte, J.; Aubert, J.; Sulk, M.; Novak, P.; Schwab, V.D.; Mess, C.; Cevikbas, F.; Rivier, M.; Carlavan, I.; et al. Clinical, cellular, and molecular aspects in the pathophysiology of rosacea. J. Investig. Dermatol. Symp. Proc. 2011, 15, 2-11. [CrossRef] [PubMed]

65. Lindow, K.B.; Warren, C. Understanding rosacea. A guide to facilitating care. Am. J. Nurs. 2001, 101, 44-51. [CrossRef] [PubMed]

66. Detmar, M. Molecular regulation of angiogenesis in the skin. J. Investig. Dermatol. 1996, 106, $207-208$. [CrossRef] [PubMed]

67. Aroni, K.; Tsagroni, E.; Kavantzas, N.; Patsouris, E.; Ioannidis, E. A study of the pathogenesis of rosacea: How angiogenesis and mast cells may participate in a complex multifactorial process. Arch. Dermatol. Res. 2008, 300, 125-131. [CrossRef] [PubMed]

68. Kim, M.; Kim, K.E.; Jung, H.Y.; Jo, H.; Jeong, S.W.; Lee, J.; Kim, C.H.; Kim, H.; Cho, D.; Park, H.J. Recombinant erythroid differentiation regulator 1 inhibits both inflammation and angiogenesis in a mouse model of rosacea. Exp. Dermatol. 2015, 24, 680-685. [CrossRef] [PubMed]

69. Park, C.C.; Morel, J.C.; Amin, M.A.; Connors, M.A.; Harlow, L.A.; Koch, A.E. Evidence of IL-18 as a novel angiogenic mediator. J. Immunol. 2001, 167, 1644-1653. [CrossRef] [PubMed]

70. Smith, J.R.; Lanier, V.B.; Braziel, R.M.; Falkenhagen, K.M.; White, C.; Rosenbaum, J.T. Expression of vascular endothelial growth factor and its receptors in rosacea. Br. J. Ophthalmol. 2007, 91, 226-229. [CrossRef] [PubMed]

71. Chajra, H.; Nadim, M.; Auriol, D.; Schweikert, K.; Lefevre, F. Combination of new multifunctional molecules for erythematotelangiectatic rosacea disorder. Clin. Cosmet. Investig. Dermatol. 2015, 8, 501-510. [CrossRef] [PubMed]

72. Domingo, D.S.; Camouse, M.M.; Hsia, A.H.; Matsui, M.; Maes, D.; Ward, N.L.; Cooper, K.D.; Baron, E.D. Anti-angiogenic effects of epigallocatechin-3-gallate in human skin. Int. J. Clin. Exp. Pathol. 2010, 3, 705-709. [PubMed]

73. Miyoshi, K.; Takaishi, M.; Nakajima, K.; Ikeda, M.; Kanda, T.; Tarutani, M.; Iiyama, T.; Asao, N.; DiGiovanni, J.; Sano, S. STAT3 as a therapeutic target for the treatment of psoriasis: A clinical feasibility study with STA-21, a STAT3 inhibitor. J. Investig. Dermatol. 2011, 131, 108-117. [CrossRef] [PubMed]

(C) 2016 by the authors; licensee MDPI, Basel, Switzerland. This article is an open access article distributed under the terms and conditions of the Creative Commons Attribution (CC-BY) license (http://creativecommons.org/licenses/by/4.0/). 